\title{
Computer Aided Modeling of Human Mastoid Cavity Biomechanics Using Finite Element Analysis
}

\author{
Chia-Fone Lee, ${ }^{1,2,3}$ Peir-Rong Chen, ${ }^{2,3}$ Wen-Jeng Lee, ${ }^{4}$ Yuan-Fang Chou, ${ }^{5}$ \\ Jyh-Horng Chen, ${ }^{1,6}$ and Tien-Chen Liu ${ }^{7}$ \\ ${ }^{1}$ Institute of Biomedical Engineering, National Taiwan University, 790 Taipei, Taiwan \\ ${ }^{2}$ Department of Otolaryngology, Buddhist Tzu Chi General Hospital, 790 Hualien, Taiwan \\ ${ }^{3}$ Department of Medicine, Tzu Chi University, 790 Hualien, Taiwan \\ ${ }^{4}$ Department of Medical Imaging, National Taiwan University Hospital, 100 Taipei, Taiwan \\ ${ }^{5}$ Institute of Mechanical Engineering, College of Mechanical Engineering, National Taiwan University, \\ 106 Taipei, Taiwan \\ ${ }^{6}$ Institute of Electrical Engineering, College of Electrical Engineering and Computer Science, National Taiwan University, \\ 106 Taipei, Taiwan \\ ${ }^{7}$ Department of Otolaryngology, National Taiwan University Hospital, 100 Taipei, Taiwan
}

Correspondence should be addressed to Tien-Chen Liu, liuent@ntu.edu.tw

Received 27 April 2009; Accepted 16 June 2009

Academic Editor: João Manuel R. S. Tavares

Copyright ( $) 2010$ Chia-Fone Lee et al. This is an open access article distributed under the Creative Commons Attribution License, which permits unrestricted use, distribution, and reproduction in any medium, provided the original work is properly cited.

\begin{abstract}
The aim of the present study was to analyze the human mastoid cavity on sound transmission using finite element method. Pressure distributions in the external ear canal and middle ear cavity at different frequencies were demonstrated. Our results showed that, first, blocking the aditus improves middle ear sound transmission in the 1500 - to $2500-\mathrm{Hz}$ range and decreases displacement in frequencies below $1000 \mathrm{~Hz}$ when compared with the normal ear. Second, at frequencies lower than $1000 \mathrm{~Hz}$, the acoustic pressures were almost uniformly distributed in the external ear canal and middle ear cavity. At high frequencies, higher than $1000 \mathrm{~Hz}$, the pressure distribution varied along the external ear canal and middle ear cavity. Third, opening the aditus, the pressures difference in $\mathrm{dB}$ between the middle ear cavity and external ear canal were larger than those of the closed mastoid cavity in low frequency $(<1000 \mathrm{~Hz})$. Finally, there was no significant difference in the acoustic pressure between the oval window and round window is noted and increased by $5 \mathrm{~dB}$ by blocking the aditus. These results suggest that our complete FE model including the mastoid cavity is potentially useful and can provide more information in the study of middle ear biomechanics.
\end{abstract}

\section{Introduction}

The human middle ear, including the tympanic membrane (TM) and the three auditory ossicles (malleus, incus, and stapes), is the mechanical system for sound transmission from the outer to the inner ear. A number of parameters such as the shape and stiffness of the TM, shape and volume of the external ear canal, and volume and pressure of the middle ear cavity directly affect acoustic-mechanical transmission through the middle ear. Changes in these parameters are often related to pathophysiologic conditions of the ear. The function of human hearing was investigated through the use of models. Among these models, the following models for the middle ear represent the state of the art in the area. In general, there are two groups of models. The first group consists of electroacoustic circuit models based on the close link between acoustics and electrical engineering [1$8]$. The second group is composed of structural mechanical models, mainly finite element (FE) models of the tympanic membrane and ossicles in humans [9-19] and in animals [20-22].

FE analysis is a computer simulation technique used in engineering and biomechanical analysis. The first FE model of the cat ear drum was reported by Funnell and Laszlo [20]. Since then, FE modeling of middle ear biomechanics has become a rapidly growing area of research. The advent 
of high-resolution computed tomography (HRCT) made it possible to perform virtual instead of physical sectioning, and computer assistance facilitated the construction of reliable three-dimensional (3D) mathematical anatomic models. Using the combined technologies of $\mathrm{FE}$ analysis and $3 \mathrm{D}$ reconstruction of the middle ear from HRCT, we developed an FE model of the human middle ear with TM, ossicular bone, middle ear ligament, and middle ear boundaries [23]. This model was validated by comparing data from it to published experimental measurements, and it was tested in several otologic applications $[24,25]$.

To date, the FE model represents the precise geometric configurations of the ossicles, TM, and ligaments/muscles and has the capability for analysis of transmission of sound through the middle ear. The FE model should, however, also include the external ear canal, middle ear cavity, and cochlea to simulate the complete acoustic-mechanical transmission in the ear. Gan et al. [26] created a two-chamber FE model (ear canal and middle ear cavity) to further simulate middle ear mechanics. They reconstructed the 3D model from a set of histological images. But the mastoid cavity was not included in their FE model, possibly due to the limited size of the histologic images. Therefore, the effects of the mastoid cavity on the sound transmission were unclear. In this paper, we report a three-chamber (ear canal, middle ear, and mastoid cavity) FE model of the right ear, incorporating middle ear ossicles, external ear canal, middle ear cavity, and mastoid cavity. The geometry and surface generation were created from HRCT images obtained in a 47-year-old man. The model was then validated by comparing the results with published experimental measurements. Acoustic-structural coupled analysis was performed to determine the function of external ear canal, middle ear cavity, and mastoid cavity for acoustic-mechanical transmission through the human middle ear.

\section{Materials and Methods}

2.1. High-Resolution Computed Tomography of Temporal Bone. In this study, HRCT scanning was performed in a 47year-old man with normal hearing and no previous otologic disorders. Otoscopic evaluation and pure tone audiometry were performed before HRCT examination. Temporal bone images obtained from the right ear were used for evaluation and reconstruction. The parameters of HRCT were described in our previous reports [23]. After capturing the images, the landmarks of the temporal bone were identified by an otolaryngologist and a radiologist (Figure 1(a)). All images were then transferred to an Amira visualization system for $3 \mathrm{D}$ reconstruction. Characteristic dimensions of the middle ear components were measured from the geometric model and compared with the published anatomic data and the data from our previous middle ear model [23].

2.2. A 3D FE Model of the Middle Ear. To prepare for FE analysis of the middle ear, the 3D model was translated into Patran (MSC Software, Perth, Australia) and ANSYS (ANSYS, Canonsburg, PA, USA): two commercially available
FE modeling packages. On the basis of the model, FE meshes of the ear were created using Patran. To facilitate the acousticstructural coupled analysis, the mesh of the FE model was slightly modified in this study. The TM was meshed using 3 layers with a total of 4293 eight-node hexahedral solid elements instead of the shell element, because the coupled analysis requires the $\mathrm{TM}$ to be a $3 \mathrm{D}$ solid structure. Using one layer of shell element with acoustic-structure interfaces on both sides does not work because each node in the shell element would have the same pressure on each side of the shell. Finally, the vibration amplitudes in a mathematical model would be more precise and reasonable than one layer. Accordingly, the tympanic annulus was meshed using 3 layers with a total of 408 eight-node hexahedral solid elements instead of shell elements. Other meshes of the FE model remained the same in our previous middle ear model [23]. The ossicles, ligaments, and tendons are considered to be isotropic materials, whereas the TM is considered to be orthotropic material. The mechanical properties of the TM, ossicles, and joints in the model (Table 1) were adopted based on the results reported by Gan et al. [26]. The structural boundaries of the middle ear include the tympanic annulus, middle ear suspensory ligament, stapedial annular ligament, and cochlear fluid. Poisson's ratio was assumed to be 0.3 for all materials of the middle ear system. The element-damping matrix for the solid elements was expressed by

$$
[C]=\alpha[M]+\beta[K],
$$

where $[M]$ and $[K]$ were element mass and stiffness matrices of the solid and shell elements, respectively, and $\alpha$ and $\beta$ were the damping parameters. The action of the cochlear fluid on the stapes footplate was modeled as a set of 49 spring-dashpot elements distributed on the footplate as our previous work [23]. The detailed modeling for the boundary conditions is shown in Table 2 [26]. Figure 1(b) shows the FE model of the human right ear including the external ear canal, ossicles with attached ligaments/muscles, and tympanic cavity. Figure 1(c) shows the extended middle ear FE model to the aditus, mastoid antrum, and mastoid cavity. Figure 1(d) shows the tympanic cavity connects to the mastoid cavity through the aditus. The tympanic cavity and mastoid cavity were displayed transparently.

The air in the external ear canal, tympanic cavity, and mastoid cavity, which enclosed the air volume $1442 \mathrm{~mm}^{3}$, $693 \mathrm{~mm}^{3}$, and $6438 \mathrm{~mm}^{3}$, respectively, was meshed with acoustic elements. The external ear canal was expressed as a bent tube with rigid walls based on the dimensions obtained through HRCT scanning. The length of canal from the umbo to the entry section along the canal axis was about $3.04 \mathrm{~cm}$ and close to result of Egolf et al. [27]. The canal length superiorly was $2.86 \mathrm{~cm}$ and the length inferiorly was $3.21 \mathrm{~cm}$. The cross-sectional area varied from $65.45 \mathrm{~mm}^{2}$ (near the TM) to $96.19 \mathrm{~mm}^{2}$ at the canal entrance. The published anatomical data for the external air volume would be ranged from 830 to $1972 \mathrm{~mm}^{3}$ [28]. A large difference in volume of the middle ear cavity 


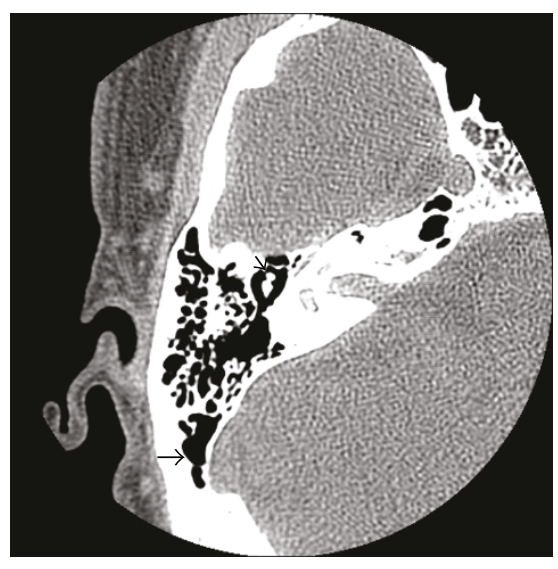

(a)

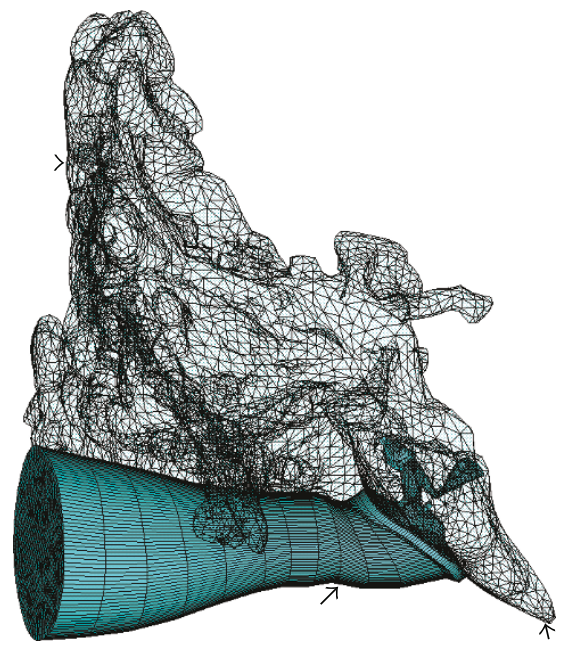

(c)

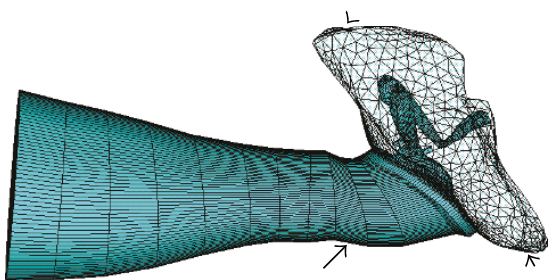

(b)

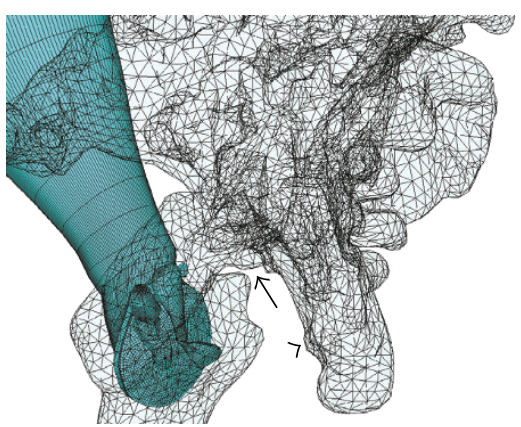

(d)

FIGURE 1: (a) Axial HRCT image of a normal right temporal bone, including ossicles (small arrow) and mastoid cavity (large arrow). (b) Finite element model of human right ear including tympanic membrane, ossicles (malleus, incus, and stapes), suspensory ligament/muscles, tympanic annulus, Eustachian tube (small arrow), external ear canal (large arrow) and tympanic cavity (arrow head) in anterior view. The tympanic cavity was expressed in transparency. (c) Finite element model of human right ear including tympanic membrane, ossicles (malleus, incus, and stapes), suspensory ligament/muscles, tympanic annulus, external ear canal (large arrow), Eustachian tube (small arrow), tympanic cavity, and mastoid cavity (arrow head) in anterior view. The middle ear cavity was expressed in transparency. (d) Finite element model of human right ear demonstrated the tympanic cavity was connected to the mastoid cavity (arrow head) through the aditus (arrow).

exists between individual subjects; this volume varies from 2000 to $22000 \mathrm{~mm}^{3}$ [29]. The volume of tympanic cavity, however, is within the range of approximately $500-1000 \mathrm{~mm}^{3}$ [30]. The volume of middle ear cavity is also within the range.

2.3. FE Analysis. The acoustic analysis in ANSYS (ANSYS Inc., Canonsburg, PA) programs only involves modeling the fluid medium and the surrounding structure [31]. A coupled acoustic analysis takes the fluid-structure interaction into account. The acoustic pressure in the fluid medium is determined by the wave equation. The interaction of the fluid and the structure at a mesh interface caused the acoustic pressure to exert a force applied to the structure and the structure motion produces an effective fluid load.
The governing finite element matrix equations produce the following:

$$
\left[\begin{array}{cc}
M_{S} & 0 \\
\rho_{0} R^{T} & M_{f}
\end{array}\right]\left[\begin{array}{c}
\ddot{u} \\
\ddot{p}
\end{array}\right]+\left[\begin{array}{cc}
K_{s} & -R \\
0 & K_{f}
\end{array}\right]\left[\begin{array}{l}
u \\
p
\end{array}\right]=\left[\begin{array}{c}
F_{s} \\
F_{f}
\end{array}\right] ;
$$

$p$ and $u$ are the fluid pressure and the structure displacement, respectively. $M_{S}$ is the structure mass matrix. $K_{S}$ is the structure stiffness matrix. Correspondingly, $M_{f}$ is the fluid mass matrix, $\rho_{0}$ is the fluid density and $K_{f}$ is the fluid stiffness matrix. $F_{f}$ is the applied fluid pressure vector at the interface obtained by integrating the pressure over the area of the surface. $R$ is a coupling matrix that represents the effective surface area associated with each node on the fluidstructure interface (FSI). Both the structural and acoustic load quantities that are produced at the acoustic-structure 
TABLE 1: Mechanical properties used for the middle ear finite element model.

\begin{tabular}{|c|c|}
\hline Structure & Data for the finite element model \\
\hline \multicolumn{2}{|l|}{ Eardrum } \\
\hline Density & $1.2 \mathrm{~g} / \mathrm{cm}^{3}$ \\
\hline Young's modulus (pars flaccida) & $1.0 \times 10^{7} \mathrm{~N} / \mathrm{m}^{2}$ \\
\hline (pars tensa $)$ & $\begin{array}{c}2.0 \times 10^{7} \mathrm{~N} / \mathrm{m}^{2} \text { (longitudinal direction) } \\
3.5 \times 10^{7} \mathrm{~N} / \mathrm{m}^{2} \text { (radial direction) }\end{array}$ \\
\hline Thickness & $0.1 \mathrm{~mm}$ \\
\hline \multicolumn{2}{|l|}{ Malleus } \\
\hline \multicolumn{2}{|l|}{ Density } \\
\hline Head & $2.55 \times 10^{3} \mathrm{Kg} / \mathrm{m}^{3}$ \\
\hline Neck & $4.53 \times 10^{3} \mathrm{Kg} / \mathrm{m}^{3}$ \\
\hline Handle & $3.70 \times 10^{3} \mathrm{Kg} / \mathrm{m}^{3}$ \\
\hline Young's modulus & $1.41 \times 10^{10} \mathrm{Kg} / \mathrm{m}^{2}$ \\
\hline \multicolumn{2}{|l|}{ Incus } \\
\hline \multicolumn{2}{|l|}{ Density } \\
\hline Body & $2.36 \times 10^{3} \mathrm{Kg} / \mathrm{m}^{3}$ \\
\hline Short process & $2.26 \times 10^{3} \mathrm{Kg} / \mathrm{m}^{3}$ \\
\hline Long process & $5.08 \times 10^{3} \mathrm{Kg} / \mathrm{m}^{3}$ \\
\hline Young's modulus & $1.41 \times 10^{10} \mathrm{~N} / \mathrm{m}^{2}$ \\
\hline \multicolumn{2}{|l|}{ Stapes } \\
\hline Density & $2.20 \times 10^{3} \mathrm{Kg} / \mathrm{m}^{3}$ \\
\hline Young's modulus & $1.41 \times 10^{10} \mathrm{~N} / \mathrm{m}^{2}$ \\
\hline
\end{tabular}

TABLE 2: Structure boundary conditions used for the middle ear finite element model.

\begin{tabular}{lcc}
\hline Middle ear components & Young's modulus or spring constant & Damping \\
\hline Superior mallear ligament & $4.9 \times 10^{6} \mathrm{~N} / \mathrm{m}^{2}$ & $\beta=0.0001 \mathrm{~s}$ \\
Lateral mallear ligament & $6.7 \times 10^{6} \mathrm{~N} / \mathrm{m}^{2}$ & $6.5 \times 10^{6} \mathrm{~N} / \mathrm{m}^{2}$ \\
Posterior incudal ligament & $2.1 \times 10^{7} \mathrm{~N} / \mathrm{m}^{2}$ \\
Anterior mallear ligament & $5.2 \times 10^{7} \mathrm{~N} / \mathrm{m}^{2}$ \\
Posterior stapedial muscle & $7.0 \times 10^{7} \mathrm{~N} / \mathrm{m}^{2}$ & $0.06 \mathrm{~N} \mathrm{~s} / \mathrm{m}$ \\
Tensor tympani muscle & $60 \mathrm{~N} / \mathrm{m}$ & $0 \mathrm{~N} \mathrm{~s} / \mathrm{m}$ \\
Cochlear fluid & $9 \mathrm{~N} / \mathrm{m}$ & \\
Stapedial annular ligament &
\end{tabular}

interface are functions of unknown nodal degree of freedom. Equation (2) implies that nodes on a fluid-structure interface have both displacement and pressure degree of freedom. The coupling matrix $R$ also takes into account the direction of the normal vector defined for each pair of coincident acoustic and structural element faces that comprise the interface surface. The ear consists of solid structure and acoustic media that belong to different engineering disciplines and result in different boundary conditions. The air in the external ear canal and inside the middle ear cavity was modeled as acoustic elements and governed by an acoustic wave equation under the assumptions that the fluid is compressible and inviscid with uniform mean density and pressure:

$$
\frac{1}{C^{2}} \frac{\partial^{2} p}{\partial t^{2}}=\nabla^{2} p
$$

where $p$ is acoustic pressure, $c$ is the speed of sound, and $c=$ $\sqrt{k / \rho_{0}}$ is the fluid medium, $\rho_{0}$ the mean fluid density, $k$ the bulk modulus of fluid, and $t$ is the time. The speed of sound and density of the air were assumed as $343 \mathrm{~m} / \mathrm{s}$ and $1.2 \mathrm{~kg} / \mathrm{m}^{3}$, respectively. The acoustic absorption coefficient of FSI $(\mu)$ is defined as the fraction of absorbed acoustic energy to total incident energy $[32,33]$. The absorption coefficient values are: 0.007 (TM), 0.02 (canal wall), 0.04 (cavity wall), 0.04 (ossicles), and 0.02 (ligament/muscles), respectively, [26].

2.4. Validation of the FE Model. The FE model was first tested and validated by comparing the responses of the middle ear system to harmonic pressure on the lateral surface of the TM between the FE analysis and published experimental measurements. Applied $120 \mathrm{~dB}$ SPL $(20 \mathrm{~Pa})$ to the canal was the same as McElveen's experiments, the harmonic 
analysis was conducted on the model over a frequency range of 200-8000 by using ANSYS. McElveen et al. [34] conducted a total 6 temporal bone experiments to study the effect of mastoid cavity modification on middle ear sound transmission. Measurements of umbo displacement were made at $200 \mathrm{~Hz}$ intervals from 500 to $7000 \mathrm{~Hz}$ at the TM. After the initial baseline umbo displacement measurements, the aditus and antrum were blocked with a saline-filled balloon (Fogarty catheter) inserted through a hole in the tegmen made prior to taking the measurements and closed with clay. The balloon was inflated, the hole on the tegmen was closed with clay and the measurement was repeated. Peak-to peak umbo displacement, aditus open versus closed in McElveen's human temporal bone 3 was used for model validation.

\section{Results}

The umbo and predicted stapes footplate displacements while the aditus was open or blocked were converted to the frequency response curve of peak-to-peak displacement. Figure 2(a) contains both simulated umbo displacement and McElveen's data [34]. The predicted stapes footplate displacements are shown in Figure 2(b). Blocking the aditus decreases displacement in the low frequencies below $1000 \mathrm{~Hz}$. Displacement in the mid-frequencies, 1500 and $2500 \mathrm{~Hz}$, was increased by aditus blockage. The FE result showed a peak increase of vibration amplitude at an approximate frequency of $4000 \mathrm{~Hz}$, with the amplitude response pattern similar to the experimental results and smoother than the experimental results. The effects of aditus blockage are small in this model. The effects might come from different volume sizes of mastoid cavity.

Figure 3(a) shows the FE model-predicted frequency response curves of relative acoustic pressure at several different locations in the canal and middle ear cavity (open the aditus) when a harmonic sound pressure of $120 \mathrm{~dB}$ SPL was applied at the canal entrance with the ear canal open. The position of measuring pressures in mastoid cavity is located in the central part and about $3.8 \mathrm{~cm}$ from the oval window, $4.2 \mathrm{~cm}$ from the round window and $3.1 \mathrm{~cm}$ from the incudostapedial joint. Likewise, Figure 3(b) demonstrates the FE model-predicted frequency response curves of relative acoustic pressure at several different locations in the canal and closed mastoid (blocking the aditus) cavity when a harmonic sound pressure of $120 \mathrm{~dB}$ SPL was applied at the canal entrance with the ear canal open. The FE result shows an 18-dB SPL increase of sound pressure around a frequency of $4000 \mathrm{~Hz}$ with a pressure response pattern similar to the experimental results. The small-dotted line in the figure is the experimental curve obtained by Shaw [35]. When the aditus was blocked (Figure 3(b)), the FE results showed that the pressure responses at four locations in the cavity were almost the same at frequencies below $4000 \mathrm{~Hz}$. At frequencies higher than $4000 \mathrm{~Hz}$, the difference of magnitude was within $5 \mathrm{~dB}$. With open aditus (Figure 3(a)), the FE results showed that the pressure responses at five locations in the cavity were almost identical at frequencies below $700 \mathrm{~Hz}$. At frequencies higher than $700 \mathrm{~Hz}$, the pressure difference in $\mathrm{dB}$ at the mastoid cavity was smaller than at the tympanic cavity. With open aditus, the pressure difference in $\mathrm{dB}$ differences between the canal entrance and middle ear cavity were larger than those of the closed cavity in low frequency $(<1000 \mathrm{~Hz})$. The FE acoustic pressure distributions in the ear canal and middle ear cavity were obtained at frequencies of 1000 and $8000 \mathrm{~Hz}$, respectively, when a sound pressure of $120 \mathrm{~dB}$ SPL was delivered at the canal entrance (Figure 4). These results showed that acoustic pressure distributions in external ear canal and middle ear cavity were functions of frequency. At frequencies lower than $1000 \mathrm{~Hz}$, the acoustic pressures were almost uniformly distributed in the external ear canal. At frequencies higher than $1000 \mathrm{~Hz}$, the pressure distribution varied along the external ear canal and middle ear cavity. To demonstrate the pressure distribution in the middle ear cavity, we hid the external ear canal from the FE model and rescaled the magnitude bar (Figure 5). Likewise, the pressure distribution varied along the middle ear cavity.

\section{Discussion}

This model is the first one characterized by accurate structural dimensional and geometric shapes of middle ear structures, external ear canal, and mastoid cavity in the human. To confirm the validity of this model, the vibration amplitude of the umbo obtained with this model was compared with that derived from experimental measurement data. The predicted vibration amplitude of stapes was also shown. It was difficult to measure stapes vibration amplitude without opening the middle ear cavity. If a complete FE model of the middle ear were constructed, spatial variations in displacement on the TM, three ossicular vibrations, and spatial pressure distributions in the middle ear cavity and external ear canal could clarified without direct measurement, which are difficult to perform. It appeared that the results from the temporal bone experiments and the FE-predicted results match, namely that blocking the aditus improves middle ear sound transmission in the 1500 to $2500 \mathrm{~Hz}$ range and decreases displacement in the low frequencies below $1000 \mathrm{~Hz}$ when compared with the normal ear. Blocking the aditus eliminates the compliance of the mastoid cavity thus stiffening the TM and decreasing low frequency transmission, while opening the aditus increases middle ear cavity compliance, decrease TM stiffness and improves the low-frequency response. It has been reported that the effect of the mastoid cavity on the vibration of the TM is remarkable at low frequency and that it behaves like a spring $[17,30,36]$. The mastoid cavity would enhance sound transmission at low frequency $(<1000 \mathrm{~Hz})$ because the spring constant of the air in the mastoid cavity is inversely proportional to its volume. In McElveen's experimental results [34], blocking the aditus decreased transmission in two bones and increased transmission in one bone. The effects were small between results. Because of the small numbers of bone studies and the individual variations between bones, any conclusion about the clinical significance of the temporal bone results might be cautiously. The real 


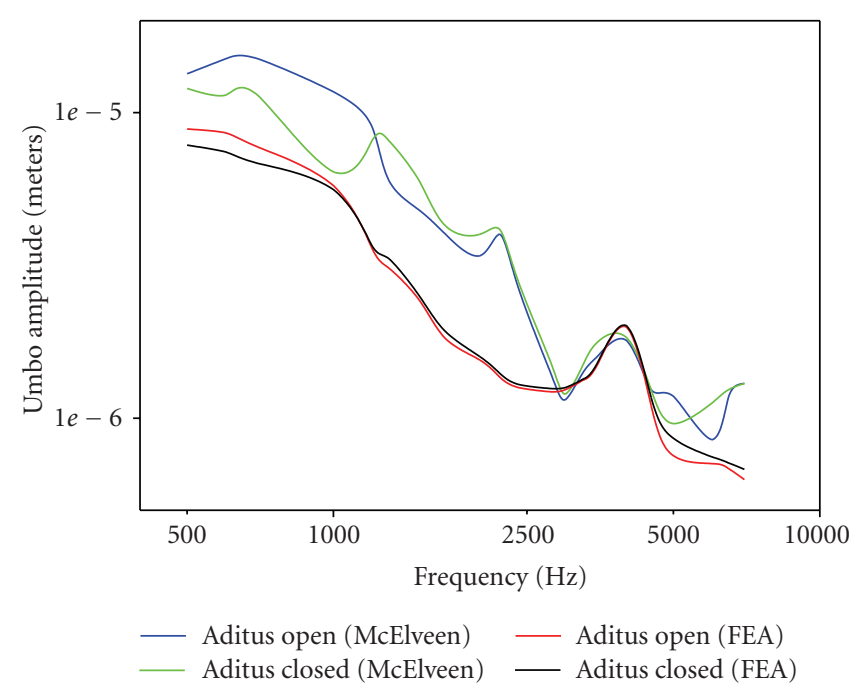

(a)

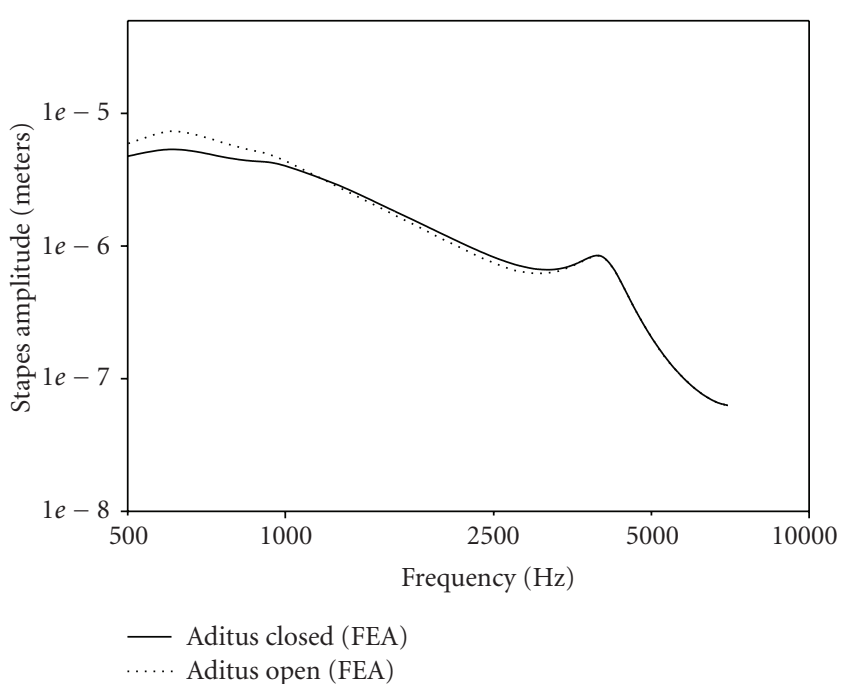

(b)

Figure 2: (a) Amplitude of umbo displacement versus frequency of the acoustic stimuli at $120 \mathrm{~dB}$ SPL (aditus open versus aditus closed) in the ear canal. The finite element model predicted umbo displacements were close to McElveen's experimental results. (b) Amplitude of predicted stapes footplate displacement versus frequency of the acoustic stimuli at $120 \mathrm{~dB}$ SPL (aditus open versus aditus closed).

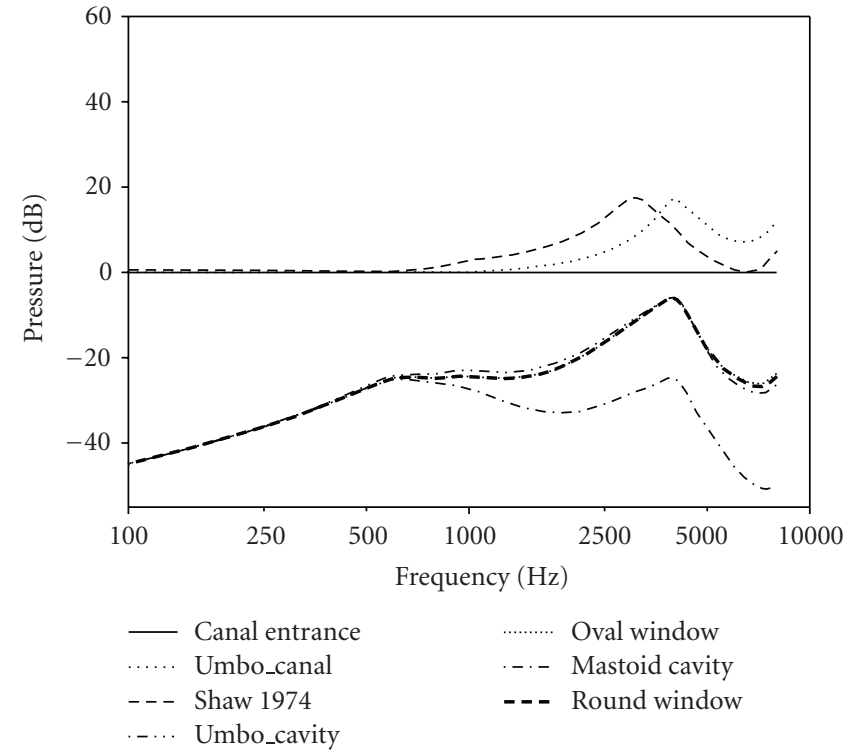

(a)

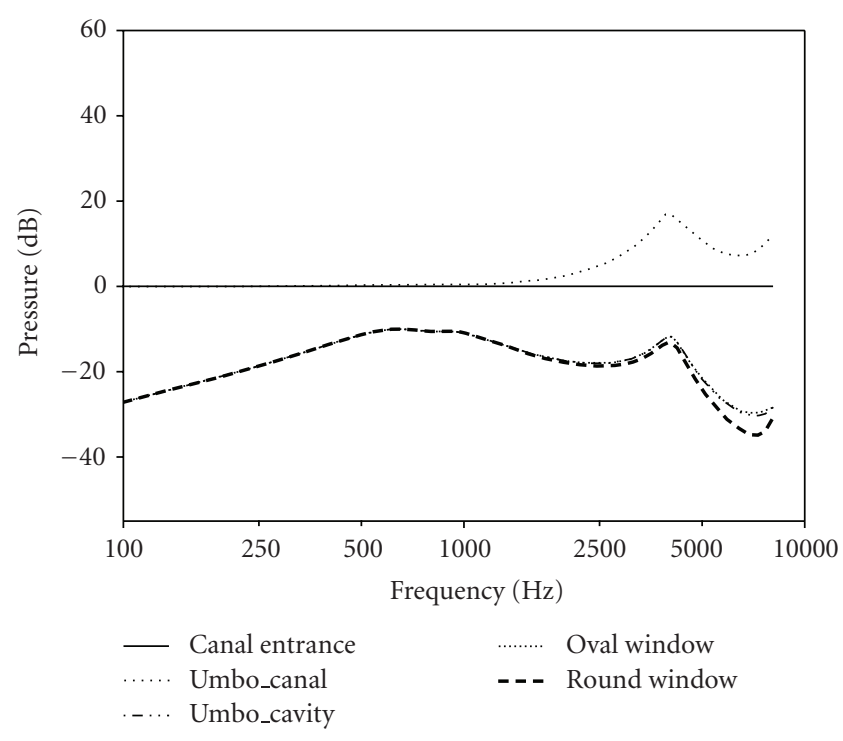

(b)

Figure 3: (a) FE frequency response curves of harmonic sound pressure at five locations inside middle ear cavity in the canal when aditus open. The $120 \mathrm{~dB}$ SPL was input at the canal entrance. The pressure magnitude was relative to the canal entrance. (b) FE frequency response curves of harmonic sound pressure at four locations inside middle ear cavity in the canal when aditus closed. The $120 \mathrm{~dB}$ SPL was input at the canal entrance. The pressure magnitude was relative to the canal entrance.

results could be affected by confounding variables including middle ear injury, stiffness of TM, and the mobility status of the ossicular chains. Therefore, more large numbers of temporal bone studies should be needed. Some small peaks in umbo displacement were noted in McElveen's results. According to umbo and stapes displacement measurements in temporal bones and living humans, in some $30 \%$ of ears, the tympanic membrane (TM) does not produce a smooth frequency response over the important hearing frequencies [37]. Goode [38] reported that measurements of umbo displacement for a constant sound pressure level (SPL) at the TM in 22 frequencies between 200 and $6000 \mathrm{~Hz}$ showed peaks and valleys of more than $10 \mathrm{~dB}$. This is possibly the result of previous injuries, both major and minor, to the TM, and perhaps to the ossicles [38]. Our FE model curve is lower than the experimental curve; however, the trend was similar 


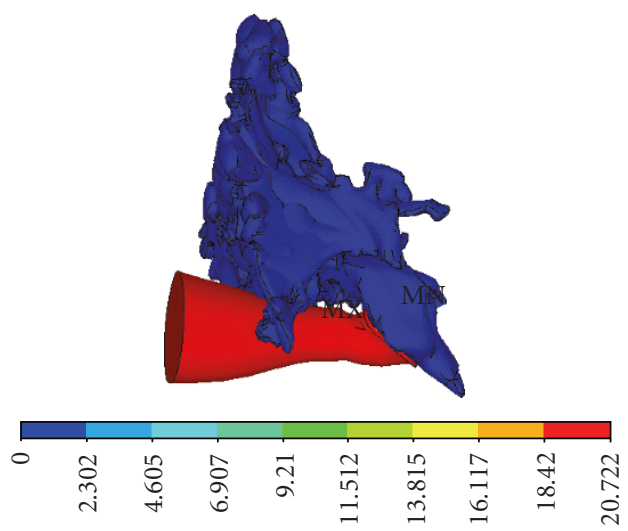

(a)

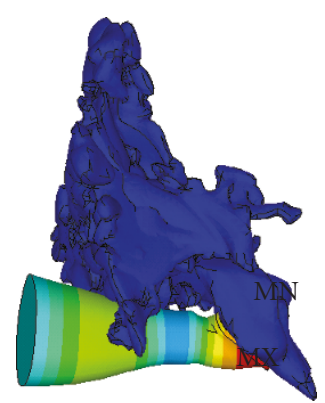

\begin{tabular}{|c|c|c|c|c|c|c|c|c|c|c|}
\hline $\begin{array}{l}\Re \\
\infty \\
a\end{array}$ & $\begin{array}{l}\infty \\
\stackrel{\infty}{ก} \\
\stackrel{2}{n}\end{array}$ & 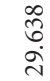 & $\begin{array}{l}\bar{n} \\
\text { ñ } \\
\text { mे }\end{array}$ & & 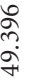 & & 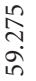 & & $\begin{array}{l}\text { 苟 } \\
\text { ğ }\end{array}$ & \\
\hline
\end{tabular}

(b)

FIGURE 4: (a) Acoustic pressure distribution in the external ear canal and middle ear cavity predicted by the FE model at frequency of $1000 \mathrm{~Hz}$. The sound pressure applied at the canal entrance was $120 \mathrm{~dB}$ SPL (20 Pa). The color bands represent different pressure levels relative to the canal entrance pressure. (b) Acoustic pressure distribution in the external ear canal and middle ear cavity predicted by the FE model at frequency of $8000 \mathrm{~Hz}$. The sound pressure applied at the canal entrance was $120 \mathrm{~dB}$ SPL (20 Pa). The color bands represent different pressure levels relative to the canal entrance pressure.

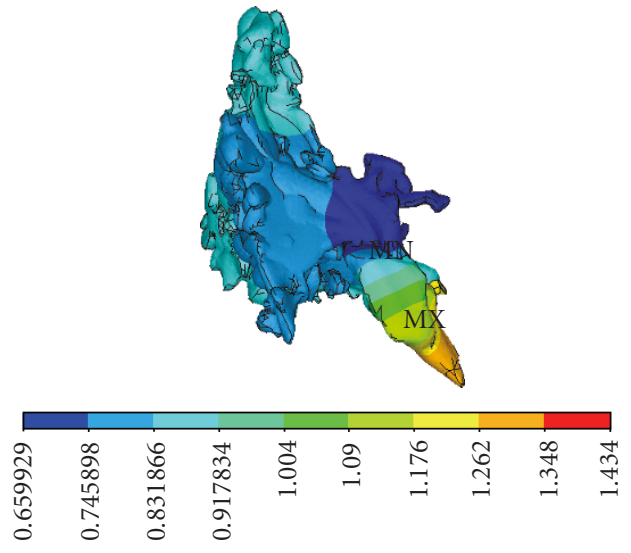

(a)
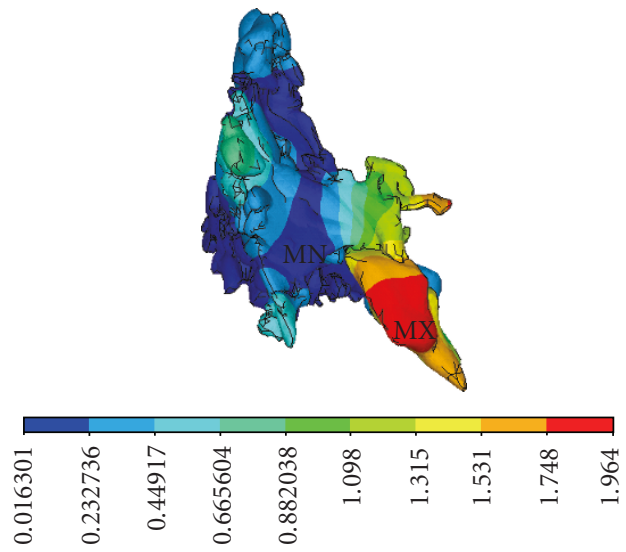

(b)

Figure 5: (a) Acoustic pressure distribution in middle ear cavity predicted by the FE model at frequency of $1000 \mathrm{~Hz}$. The sound pressure applied at the canal entrance was $120 \mathrm{~dB}$ SPL $(20 \mathrm{~Pa})$. The color bands represent different pressure levels relative to the canal entrance pressure. The external ear canal was hidden. (b) Acoustic pressure distribution in middle ear cavity predicted by the FE model at frequency of $8000 \mathrm{~Hz}$. The sound pressure applied at the canal entrance was $120 \mathrm{~dB}$ SPL $(20 \mathrm{~Pa})$. The color bands represent different pressure levels relative to the canal entrance pressure. The external ear canal was hidden.

to the experimental curve. The difference between the FE model and the experimental data may also result from the variations of individual temporal bone.

The acoustic pressure distributions in the external ear canal and middle ear cavity are spatially visualized and quantified by our FE model. Our result is the complete FE model, including the external ear canal, TM, ossicles, ligaments/muscles, tympanic cavity, and mastoid cavity. The results demonstrated that acoustic pressure distributions in ear canal and middle ear cavity are functions of frequency and different pressure measurement locations (Figure 3). The difference of acoustic pressure between the ear canal and middle ear cavity was caused by high acoustic impedance of the TM that was induced by attached middle ear and inner ear structure. In the FE coupled analysis, the mastoid cavity effect was taken into the consideration for acoustic impedance. The air vibration in the middle ear cavity was lower than the air vibration in the canal. At low frequencies $(f<1000 \mathrm{~Hz})$, the acoustic pressure was uniformly distributed in the ear canal (Figure 4(a)). At high frequencies $(f>1000 \mathrm{~Hz})$, the pressure distribution varied along the canal (Figure 4(b)). The results reflect superposition of the incident and reflected sound wave from the TM and canal wall in the canal. The sound pressure difference in $\mathrm{dB}$ in the middle ear cavity is expected to vary with the air volume of the cavity (Figure 5). The acoustic pressure in the closed mastoid cavity is $10-25 \mathrm{~dB}$ lower than that of the canal entrance over the frequency range of $100-8000 \mathrm{~Hz}$. 
With open aditus, the acoustic pressure in the middle ear cavity is $10-45 \mathrm{~dB}$ lower than that of the canal entrance over the frequency range of $100-8000 \mathrm{~Hz}$. This big drop of acoustic pressure in the cavity is caused by the high acoustic impedance of the TM induced by the attached middle ear and inner ear structure.

There was no significant difference of the acoustic pressure measured at different locations in the middle ear cavity at low frequency. As frequency increases, the pressure difference in $\mathrm{dB}$ between the oval window and round window is noted and increased by less than $5 \mathrm{~dB}$. These results demonstrate that window pressure difference of the acoustic pathway for sound transmission to the inner ear is insignificant. The same conclusion is also obtained from experimental measurements on the temporal bone by Voss et al. [30], Peake et al. [4], and FE results by Gan et al. [26].

In conclusion, we created an FE model that not only includes the external ear canal and tympanic cavity but also the mastoid cavity, which can help us to understand the mastoid cavity effect on sound transmission. Tympanomastoid surgery modifies the middle ear cavity in various ways. These modifications might have important effects on sound transmission of the middle ear [34]. The acoustic effects of cavity modification by different types of tympanoplasty and mastoidectomy are difficult to determine clinically because TM and ossicular reconstruction are often undertaken as well. These results suggest that the FE model is potentially useful in the study of middle ear biomechanics and in the design and testing of the implantable middle ear hearing devices [39]. It would be possible to predict how middle ear function is affected by various kinds of middle ear pathologies and to understand how individual differences in middle ear structures affect that function prior to surgery. The model could be further improved in several aspects as finding more accurate boundary conditions and adding the structure of cochlea and the cochlear fluid into the model [40]. The overall thickness of TM $(0.1 \mathrm{~mm})$ was adopted in our model. Fay et al. [19] incorporates the measurement of the geometry of the ear canal, the 3D asymmetrical geometry of the eardrum and the details of the eardrum fiber structure. To develop a more comprehensive 3D FE model of human ear for multi-field FE analysis using detail TM structures and coupling the current FE model is our next goals. In addition, ligaments/tendons have a clear different behavior in tension and compression, in fact, stiffness in tension is much higher than in compression. The ligaments/tendons in the middle ear were traction free and essentially onedirection member. The behavior was dominant in axial direction. Therefore, if we chose the proper values, the hypothesis of isotropic behavior can be appropriated. A variety of mechanical tests have been reported to measure properties of soft tissue, such as uniaxial tensile, strip biaxial tension, and shear tests. In addition to experimental measurement, numerous material models have been developed to simulate the behavior of tissue in analytical ways [41]. Weiss et al. [42] used a hyperelastic material model with an exponential strain energy function to fit experimental curves of human medial collateral ligament through FEA. There are several nonlinear hyperelastic material models available for analyzing mechanical properties of biological soft tissue, such as the Ogden, Mooney-Rivlin and Yeoh models. In the future, these methods can be used to improve our FE model of human ear. The further study will focus on how the alteration in structure, pathology, collagen fiber layer in tympanic membrane and different air volume sizes of mastoid cavity would affect the acoustic-mechanical transmission through the ear canal and middle ear to the inner ear.

\section{Acknowledgments}

This work was supported by a grant from the National Taiwan University Hospital to T.C.L. (Grant no. NTUH 96A01) and a grant from the Buddhist Tzu Chi General Hospital to C.F.L. (Grant no. TCRD 9703, 9704, 9801, and 9802).

\section{References}

[1] Y. Onchi, "Mechanism of middle ear," Journal of the Acoustical Society of America, vol. 33, p. 794, 1961.

[2] J. Zwislocki, "Analysis of middle ear function. I. Input impedance," Journal of the Acoustical Society of America, vol. 34, pp. 1514-1523, 1962.

[3] C. A. Shera and G. Zweig, "Phenomenological characterization of eardrum transduction," Journal of the Acoustical Society of America, vol. 90, no. 1, pp. 253-262, 1991.

[4] W. T. Peake, J. J. Rosowski, and T. J. Lynch III, "Middle-ear transmission: acoustic versus ossicular coupling in cat and human," Hearing Research, vol. 57, no. 2, pp. 245-268, 1992.

[5] R. L. Goode, M. Killion, K. Nakamura, and S. Nishihara, "New knowledge about the function of the human middle ear: development of an improved analog model," American Journal of Otology, vol. 15, no. 2, pp. 145-154, 1994.

[6] J. J. Rosowski and S. N. Merchant, "Mechanical and acoustic analysis of middle ear reconstruction," American Journal of Otology, vol. 16, no. 4, pp. 486-497, 1995.

[7] H. Hudde and C. Weistenhöfer, "A three-dimensional circuit mof the middle ear," Acustica, vol. 83, no. 3, pp. 535-549, 1997.

[8] S. Puria and J. B. Allen, "Measurements and model of the cat middle ear: evidence of tympanic membrane acoustic delay," Journal of the Acoustical Society of America, vol. 104, no. 6, pp. 3463-3481, 1998.

[9] K. R. Williams and T. H. J. Lesser, "A finite element analysis of the natural frequencies of vibration of the human tympanic membrane I," British Journal of Audiology, vol. 24, no. 5, pp. 319-327, 1990.

[10] H. Wada, T. Metoki, and T. Kobayashi, "Analysis of dynamic behavior of human middle ear using a finite-element method," Journal of the Acoustical Society of America, vol. 92, no. 6, pp. 3157-3168, 1992.

[11] A. Eiber and A. Kauf, "Berechnete Verschiebung der Mittelohrknochen unter statischer Belastung," HNO, vol. 42, no. 12, pp. 754-759, 1994.

[12] K. R. Williams, A. W. Blayney, and H.J. Rice, "Middle ear mechanics as examined by the finite element method," in Middle Ear Mechanics in Research and Otosurgery: Proceedings of the International Workshop on Middle Ear Mechanics, K. B. Hüttenbrink, Ed., pp. 40-47, 1997.

[13] H.-J. Beer, M. Bornitz, H.-J. Hardtke, et al., "Modelling of components of the human middle ear and simulation of their 
dynamic behaviour," Audiology and Neuro-Otology, vol. 4, no. 3-4, pp. 156-162, 1999.

[14] M. Bornitz, T. Zahnert, H.-J. Hardtke, and K.-B. Hüttenbrink, "Identification of parameters for the middle ear model," Audiology and Neuro-Otology, vol. 4, no. 3-4, pp. 163-169, 1999.

[15] P. J. Prendergast, P. Ferris, H. J. Rice, and A. W. Blayney, "Vibro-acoustic modelling of the outer and middle ear using the finite-element method," Audiology and Neuro-Otology, vol. 4, no. 3-4, pp. 185-191, 1999.

[16] P. Ferris and P. J. Prendergast, "Middle-ear dynamics before and after ossicular replacement," Journal of Biomechanics, vol. 33, no. 5, pp. 581-590, 2000.

[17] T. Koike, H. Wada, and T. Kobayashi, "Modeling of the human middle ear using the finite-element method," Journal of the Acoustical Society of America, vol. 111, no. 3, pp. 1306-1317, 2002.

[18] R. Z. Gan, B. Feng, and Q. Sun, "Three-dimensional finite element modeling of human ear for sound transmission," Annals of Biomedical Engineering, vol. 32, no. 6, pp. 847-859, 2004.

[19] J. Fay, S. Puria, W. F. Decraemer, and C. Steele, "Three approaches for estimating the elastic modulus of the tympanic membrane," Journal of Biomechanics, vol. 38, no. 9, pp. 1807$1815,2005$.

[20] W. R. J. Funnell and C. Laszlo, "Modeling of the cat eardrum as a thin shell using the finite-element method," Journal of the Acoustical Society of America, vol. 63, no. 5, pp. 1461-1467, 1978.

[21] W. R. Funnell, W. F. Decraemer, and S. M. Khanna, "On the damped frequency response of a finite-element model of the cat eardrum," Journal of the Acoustical Society of America, vol. 81, no. 6, pp. 1851-1859, 1987.

[22] H. M. Ladak and W. R. J. Funnell, "Finite-element modeling of the normal and surgically repaired cat middle ear," Journal of the Acoustical Society of America, vol. 100, no. 2, pp. 933-944, 1996.

[23] C.-F. Lee, P.-R. Chen, W.-J. Lee, J.-H. Chen, and T.-C. Liu, "Three-dimensional reconstruction and modeling of middle ear biomechanics by high-resolution computed tomography and finite element analysis," Laryngoscope, vol. 116, no. 5, pp. 711-716, 2006.

[24] C.-F. Lee, L.-P. Hsu, P.-R. Chen, Y.-F. Chou, J.-H. Chen, and T.-C. Liu, "Biomechanical modeling and design optimization of cartilage myringoplasty using finite element analysis," Audiology and Neurotology, vol. 11, no. 6, pp. 380-388, 2006.

[25] C.-F. Lee, J.-H. Chen, Y.-F. Chou, L.-P. Hsu, P.-R. Chen, and T.C. Liu, "Optimal graft thickness for different sizes of tympanic membrane perforation in cartilage myringoplasty: a finite element analysis," Laryngoscope, vol. 117, no. 4, pp. 725-730, 2007.

[26] R. Z. Gan, Q. Sun, B. Feng, and M. W. Wood, "Acousticstructural coupled finite element analysis for sound transmission in human ear-pressure distributions," Medical Engineering and Physics, vol. 28, no. 5, pp. 395-404, 2006.

[27] D. P. Egolf, D. K. Nelson, H. C. Howell III, and V. D. Larson, "Quantifying ear-canal geometry with multiple computerassisted tomographic scans," Journal of the Acoustical Society of America, vol. 93, no. 5, pp. 2809-2819, 1993.

[28] J. A. Donaldson and J. M. Miller, "Anatomy of the ear," in Otolaryngology: Basic Sciences and Related Disciplines, vol. 1, pp. 75-110, Saunders, Philadelphia, Pa, USA, 1973.

[29] O. I. Molvær, F. M. Vallersnes, and M. Kringlebotn, "The size of the middle ear and the mastoid air cell. System measured by an acoustic method," Acta Oto-Laryngologica, vol. 85, no. 1-2, pp. 24-32, 1978.

[30] S. E. Voss, J. J. Rosowski, S. N. Merchant, and W. T. Peake, "Acoustic responses of the human middle ear," Hearing Research, vol. 150, no. 1-2, pp. 43-69, 2000.

[31] H. Debruyne and O. Lesaint, "About the significance of PD measurements in liquids," IEEE Transactions on Dielectrics and Electrical Insulation, vol. 10, no. 3, pp. 385-392, 2003.

[32] A. D. Pierce, Acoustic-An Introduction to Its Physical Principles and Applications, McGraw-Hill, New York, NY, USA, 1981.

[33] L. E. Kinsler, A. R. Frey, A. B. Coppens, and J. V. Sanders, Fundamentals of Acoustics, John Wiley \& Sons, New York, NY, USA, 4th edition, 2002.

[34] J. T. McElveen, C. Miller, R. L. Goode, and S. A. Falk, "Effect of mastoid cavity modification on middle ear sound transmission," Annals of Otology, Rhinology and Laryngology, vol. 91, no. 5, pp. 526-532, 1982.

[35] E. N. G. Shaw, "The external ear," in Handbook of Sensory Physiology, W. D. Keidel and W. D. Nef, Eds., vol. 1, Springer, Berlin, Germany, 1974.

[36] I. Kirikae, The Structure and Function of Middle Ear, Tokyo University Press, Tokyo, Japan, 1960.

[37] R. L. Goode, K. Nakamura, and K. Gyo, "Comments on: acoustic transfer characteristics in human middle ears studied by a SQUID magnetometer method," Journal of the Acoustical Society of America, vol. 82, pp. 1646-1654, 1987.

[38] R. L. Goode, "Current status and future of implantable electromagnetic hearing aids," Otolaryngologic Clinics of North America, vol. 28, no. 1, pp. 141-146, 1995.

[39] C.-F. Lee, J.-H. Chen, Y.-F. Chou, and T.-C. Liu, "The optimal magnetic force for a novel actuator coupled to the tympanic membrane: a finite element analysis," Biomedical Engineering, vol. 19, no. 3, pp. 171-177, 2007.

[40] R. Z. Gan, B. P. Reeves, and X. Wang, "Modeling of sound transmission from ear canal to cochlea," Annals of Biomedical Engineering, vol. 35, no. 12, pp. 2180-2195, 2007.

[41] T. Cheng and R. Z. Gan, "Experimental measurement and modeling analysis on mechanical properties of tensor tympani tendon," Medical Engineering and Physics, vol. 30, no. 3, pp. 358-366, 2008.

[42] J. A. Weiss, J. C. Gardiner, and C. Bonifasi-Lista, "Ligament material behavior is nonlinear, viscoelastic and rateindependent under shear loading," Journal of Biomechanics, vol. 35, no. 7, pp. 943-950, 2002. 